\title{
Calibration for 3D Reconstruction of Thermal Images
}

Dariusz Rzeszotarski, Bogusław Więcek

Technical University of Lodz, Institute of Electronics

Lodz, Wólczańska 211/215, Poland 90-924 dariusz.rzeszotarski@p.lodz.pl, boguslaw.wiecek@p.lodz.pl

\section{Introduction}

The calibration system presented in this article enables to calculate optical parameters i.e. intrinsic and extrinsic of both thermal and visual cameras used for 3D reconstruction of thermal images. Visual cameras are in stereoscopic set and provide a pair of stereo images of the same object which are used to perform 3D reconstruction of the examined object [8]. The thermal camera provides information about temperature distribution on the surface of an examined object. In this case the term of 3D reconstruction refers to assigning to each pixel of one of the stereo images (called later reference image) a 3D coordinate in the respective camera reference frame [8]. The computed 3D coordinate is then re-projected on to the thermograph and thus to the known 3D position specific temperature is assigned. In order to remap the 3D coordinates on to thermal image it is necessary to know the position of thermal camera against visual camera and therefore a calibration of the set of the three cameras must be performed. The presented calibration system includes special calibration board (fig.1) whose characteristic points of well known position are recognizable both by thermal and visual cameras. In order to detect calibration board characteristic points' image coordinates, especially in thermal camera, a new procedure was designed.

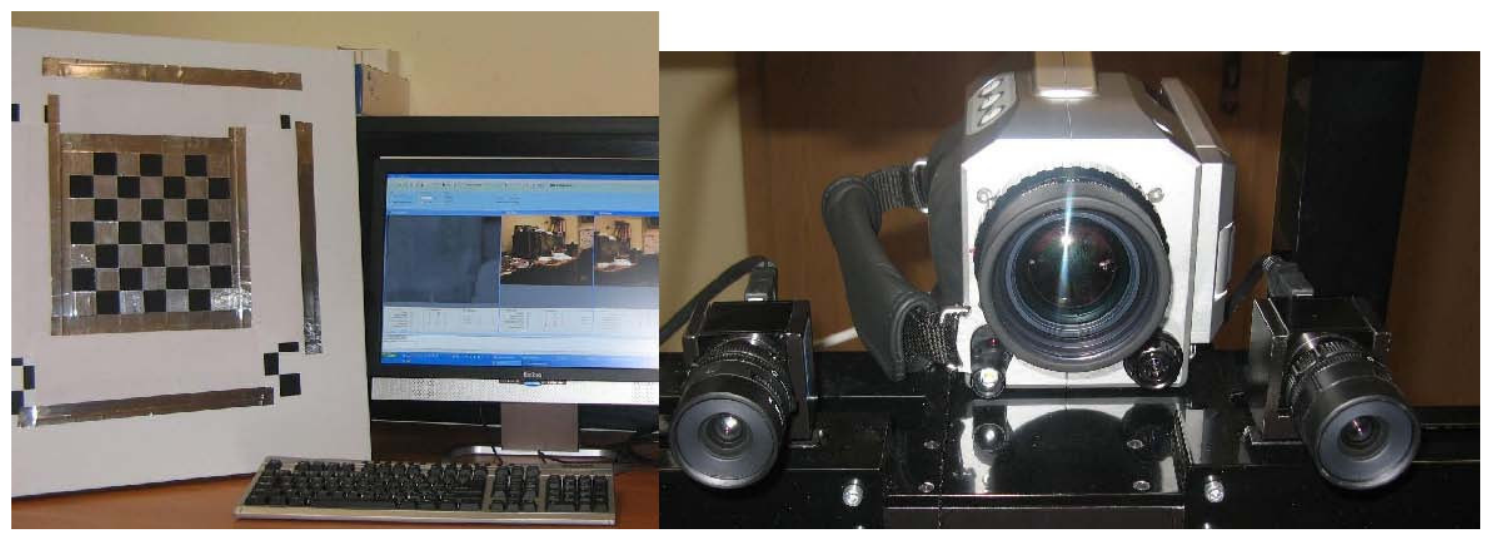

Fig. 1 Calibration Board and the set of the three cameras: thermal and visual ones.

\section{Calibration}

The calibration of depicted system includes both the calibration of stereovision pair of cameras and the calibration of thermal camera. The calibration term is meant as computing optical intrinsic and extrinsic parameters of cameras $[1,2,3]$. The extrinsic parameters define the position between respective cameras or between given camera's reference frame and another extrinsic reference frame. Extrinsic parameters of a single camera are described by translation vector $T$ and rotation $R$ matrix. The translation vector defines translation between cameras reference frame $\left(X_{c}, Y_{c}, Z_{c}\right)$ and another reference frame $(X, Y, Z)$ and the rotation matrix defines the rotations between the axes of reference frames. The relation between 3D point coordinates in the camera reference frame $P_{c}=\left[X_{c}, Y_{c}, Z_{c}\right]$ and the same $3 \mathrm{D}$ point coordinates in another reference frame $P=[X, Y, Z]$ is defined by the following formula:

$$
\mathrm{P}=\mathrm{R} \times \mathrm{P}+\mathrm{T}
$$

The beginning of the reference frame associated with single camera is presumed to be in optical center of the camera.

The intrinsic parameters are necessary to perform not only 3D reconstruction but also to correct optical distortions introduced by optical lenses. Intrinsic parameters can be divided into two groups. Parameters belonging to the first group can be confined in the so called camera matrix [3,5] and include:

- $f_{x}, f_{y}$ focal length in pixels, i.e. $f_{x}=f k_{x}$ and $f_{y}=f k_{y}, k_{x}$ is the number of pixels per millimeter in horizontal direction, $k_{y}$ is the number of pixels per millimeter in vertical direction,

- $x_{0}, y_{0}$-principal point coordinates (defined as intersection point of image plane with line perpendicular to image plane and connected with optical center of camera), 
- $y$ - coefficient defining the angle between $x$ and $y$ axes of camera image plane ( for perpendicular axes $y=0)$.

The second group of intrinsic parameters consists of coefficients depicting radial and tangential distortions caused be the camera lenses [3,5].

The calibration procedure requires a calibration object of known dimensions. In the described system a dedicated calibration board was used (printed chessboard with $7 \times 7$ square fields of $30 \times 30 \mathrm{~mm}$ each). In order to improve the visibility of chessboard fields in thermal image, the white files of calibration board were covered with aluminum foil.

The calibration procedure consists of the following steps:

- Acquisition of a few triple shots of calibration board in a few different positions seen by the three cameras; it is presumed that the external reference frame for which the rotation matrix and translation vector are computed is associated with calibration board with its beginning at the top left corner of the calibration board.

- Finding the image coordinates of chessboard corners in all the acquired triple shots; a special procedure had to be written to find characteristic points in image of calibration board in both thermal and visual cameras at the same time.

- With the known image coordinates of the chessboard corners and corresponding 3D coordinates which remain the same for each position of calibration board, as the external reference frame is associated with the board itself, the homography i.e. perspective projection matrix between calibration board plane and the image plane is calculated. For each position of calibration board the homography is found by means of maximum likelihood estimation. From thus acquired set of homographies, intrinsic parameters of cameras which remain the same for each homography are extracted first and then for each homography corresponding rotation matrix and translation vector is found.

- And last but not least the values of thus found intrinsic and extrinsic parameters are optimized with the use of gradient descent method.

\section{Experiments}

The set of three cameras was calibrated with a calibration procedure described in the previous chapter. As visual cameras in stereo set we used the digital ones, Point Grey, type Flea, of resolution 1024x768 with IEEE1394 interface [9] and with 12mm lenses; the thermal camera used in the system is the InfraTec, type VarioCam $\AA$, of $640 \times 480$ resolution. In the tables 2 and 3 the computed intrinsic and extrinsic parameters of all the three cameras are presented respectively.

Table 2. Intrinsic parameters of the three cameras

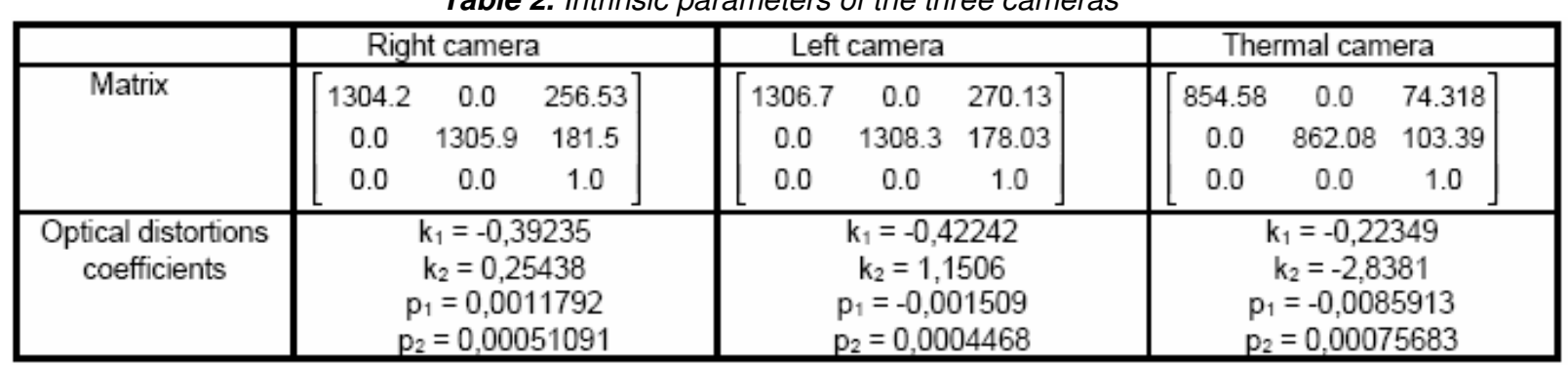

Table 3. Extrinsic parameters of set of the three cameras relating positions of left camera $R_{L R}, T_{L R}$ and thermal camera $R_{\text {THR, }} T_{\text {THR }}$ to the right (reference) one

\begin{tabular}{|c|cc|c|c|}
\hline & Left camera vs. right one & Thermal camera vs. right one \\
\hline Rotation matrix & {$\left[\begin{array}{cccc}0.99939 & 0.018628 & 0.029553 \\
-0.017941 & 0.99957 & -0.023347 \\
-0.029975 & 0.022802 & 0.99929\end{array}\right]$} & {$\left[\begin{array}{ccc}0.97079 & 0.051354 & -0.23436 \\
-0.062305 & 0.99727 & -0.03956 \\
0.23169 & 0.053006 & 0.97135\end{array}\right]$} \\
\hline Translation vector & {$\left[\begin{array}{c}-79.829 \\
-0.92904 \\
-0.23286\end{array}\right]$} & {$\left[\begin{array}{c}280.04 \\
-13.817 \\
248.34\end{array}\right]$} \\
\hline
\end{tabular}

\section{Conclusions}

The calibration system calculating intrinsic and extrinsic parameters of both thermal and visual cameras was presented. The special procedure for finding characteristic points of calibration board on thermal and visual images was designed. 


\section{BIBLIOGRAPHY}

[1] B. Cyganek, "Komputerowe przetwarzanie obrazów trójwymiarowych", Akademicka Oficyna Wydawnicza EXIT, Warszawa 2002.

[2] A. Fusiello, E. Trucco, A. Verri, "A compact algorithm for rectification of stereo pairs", Machine Vision and Applications, vol. 12, 2000, pp. 16-22.

[3] J.-Y. Bouguet, Camera Calibration Toolbox for Matlab http://www.vision.caltech.edu/bouguetj/calib_doc/index.html

[4] Open Source Computer Vision Library http://www.intel.com/technology/computing/opencv/index.htm

[5] A. Fusiello, E. Trucco, A. Verri, Rectification of Stereo Pairs http://profs.sci.univr.it/ fusiello/demo/rect/

[6] Middlebury Stereo Vision Page http://cat.middlebury.edu/stereo/

[7] Point Grey Research Inc. http://www.ptgrey.com/

[8] Rzeszotarski Darius, Więcek Bogusław: "3D Reconstruction of thermal scene with use of stereovision system", $15^{\text {th }}$ International Conference on Thermal Engineering and Thermography (THERMO). Budapest Hungary, 26-29.06.2007. 
http://dx.doi.org/10.21611/qirt.2008.14_03_04 